\title{
Lattice-Based Group Signature Scheme with Verifier-Local Revocation
}

\author{
Adeline Langlois ${ }^{1}$, San Ling ${ }^{2}$, Khoa Nguyen ${ }^{2}$, and Huaxiong Wang ${ }^{2}$ \\ 1 École Normale Supérieure de Lyon, \\ LIP (U. Lyon, CNRS, ENSL, INRIA, UCBL), \\ 46 Allée d'Italie, 69364 Lyon Cedex 07, France \\ adeline.langlois@ens-lyon.fr \\ 2 Division of Mathematical Sciences, \\ School of Physical and Mathematical Sciences, \\ Nanyang Technological University, Singapore \\ \{lingsan, khoantt, hxwang\}@ntu.edu.sg
}

\begin{abstract}
Support of membership revocation is a desirable functionality for any group signature scheme. Among the known revocation approaches, verifier-local revocation (VLR) seems to be the most flexible one, because it only requires the verifiers to possess some up-to-date revocation information, but not the signers. All of the contemporary VLR group signatures operate in the bilinear map setting, and all of them will be insecure once quantum computers become a reality. In this work, we introduce the first lattice-based VLR group signature, and thus, the first such scheme that is believed to be quantum-resistant. In comparison with existing lattice-based group signatures, our scheme has several noticeable advantages: support of membership revocation, logarithmicsize signatures, and weaker security assumption. In the random oracle model, our scheme is proved to be secure based on the hardness of the SIVP $_{\widetilde{\mathcal{O}}\left(n^{1.5}\right)}$ problem in general lattices - an assumption that is as weak as those of state-of-the-art lattice-based standard signatures. Moreover, our construction works without relying on encryption schemes, which is an intriguing feature for group signatures.
\end{abstract}

Keywords: group signature, verifier-local revocation, lattice-based cryptography.

\section{Introduction}

Group Signatures. Group signatures have been an important research topic in public-key cryptography since their introduction by Chaum and van Heyst [15]. In these schemes, all the potential signers form a group, where each signer can anonymously issue a signature on behalf of the whole group (anonymity). On the other hand, in cases of disputes, there is a tracing mechanism which can link a given signature to the identity of the misbehaving member (traceability). These two attractive features allow group signatures to find applications in various real-life scenarios, such as anonymous online communications, digital right 
management, e-commerce systems, and much more. Over the last two decades, many group signature schemes with different security models, different levels of efficiency and functionality have been proposed ([16 4 5| $8996|20| 25], \ldots)$.

One desirable functionality of group signatures is the support for membership revocation. For example, misbehaving members who issue signatures for documents, which they are not allowed to sign, should be revoked from the group. In these cases, if a group signature scheme does not support revocation, then the whole system has to be re-initialized, which is obviously an unsuitable solution in practice. Currently there are two main revocation approaches for group signatures. The first approach requires all the unrevoked members to update their signing keys after each revocation $(412811, \ldots)$. At the same time, all the signature verifiers need to download the up-to-date group public key. As a consequence, it is sometimes inconvenient to practically implement such schemes. The second approach, that is group signatures with verifier-local revocation (VLR), only requires the verifiers to possess some up-to-date revocation information, but not the signers. Since in most of real-life scenarios, the number of signature verifiers is much smaller than the number of signers, this revocation approach is more flexible and more practical. Moreover, it is akin to that of the traditional Public Key Infrastructures, where the verifiers use the latest Certificate Revocation List to check the public key of the signer. The notion of VLR group signatures was considered by Brickell [10 and Kiayias et al. 22, then formalized by Boneh and Shacham 9], further investigated and extended by Nakanishi and Funabiki 3334, Libert and Vergnaud [26, and Bichsel et al. [7. It is worth mentioning that all the existing VLR group signatures scheme operate in the bilinear map setting. Furthermore, all these schemes will be insecure once quantum computers become a reality [39]. Thus, constructing a VLR group signature schemes which is secure against quantum computers, or even outside of the bilinear map setting, is a challenging open question.

Lattice-Based Group Signatures. Lattice-based cryptography is currently considered as the most promising candidate for post-quantum cryptography. As opposed to classical cryptography (i.e., based on the hardness of factoring or discrete log problems), lattice-based cryptography is widely believed to be resistant against quantum computers, moreover, it enjoys provable security under worstcase hardness assumptions ([1/37/8 31]). Designing secure and efficient latticebased cryptographic constructions (and group signatures, in particular) becomes an intriguing challenge for the research community looking forward to the future. To the best of our knowledge, three lattice-based group signature schemes have been proposed, but none of them supports membership revocation. The first one was introduced by Gordon et al. [19] in 2010. While their scheme is of great theoretical interest, its signatures have size $\mathcal{O}(N)$, where $N$ is the number of group users. In terms of efficiency, this is a noticeable disadvantage if the group is large, e.g., group of all employees of a big company. Camenisch et al. 13 later proposed lattice-based anonymous attribute tokens system, a primitive that can be considered as a generalization of group signature. However, in their construction, the signatures size is still linear in $N$. Recently, Laguillaumie et al. [23] designed a 
scheme featuring signature size $\widetilde{\mathcal{O}}(\log N)$, which is the first lattice-based group signature that overcomes the linear-size barrier. We remark that all the above mentioned schemes follow the traditional sign-and-encrypt-and-prove paradigm: to enable the tracing mechanism, these schemes require the signer to encrypt some private information via certain type of encryption based on the Learning With Errors (LWE) problem, and then generate a sophisticated proof to prove particularly that the ciphertext is well-formed. Relying on encryption to construct group signatures may imply two troublesome issues: firstly, it makes the construction less efficient; secondly, since the whole system is secure only if the underlying encryption scheme is secure, it usually leads to a relatively strong security assumption. In particular, the recent scheme by Laguillaumie et al. 23. is only provably secure if there is no quantum algorithm to approximate the Shortest Independent Vectors Problem $\left(\operatorname{SIVP}_{\gamma}\right)$ on lattices of dimension $n$ to within certain $\gamma=\widetilde{\mathcal{O}}\left(n^{8.5}\right)$. This yields several interesting open questions in this direction: Is it possible to construct a scheme that supports membership revocation? Can lattice-based group signature schemes be free of LWE-based encryptions? How to design a more efficient scheme based on weaker security assumption?

Our Contributions. In the present work, we reply to all the above open questions positively. In particular, we introduce the first group signature with verifierlocal revocation from lattice assumptions, and thus, the first such scheme that is believed to be quantum-resistant. In comparison with known lattice-based group signatures, while the schemes from [19], 13] and 23] follow the CPA-anonymity and CCA-anonymity notions from [85, our construction satisfies the (weaker) notion of selfless-anonymity for VLR group signatures from [9] Nevertheless, our scheme has several remarkable advantages over the contemporary counterparts:

1. Functionality: Our scheme is the first lattice-based group signature that supports membership revocation. As discussed above, this is a desirable functionality for any group signature scheme.

2. Simplicity: Our scheme is conceptually very simple. The signature is basically an all-in-one proof of knowledge, made non-interactive using Fiat-Shamir paradigm [17. Moreover, the scheme departs from the traditional paradigm, and is free of LWE-based encryptions.

3. Efficiency: For a security parameter $n$ and for a group of $N$ members, the group public key and the signature have bit-sizes $\widetilde{\mathcal{O}}\left(n^{2}\right) \cdot \log N$ and $\widetilde{\mathcal{O}}(n)$. $\log N$, respectively. This result is comparable to that of [23], and is a noticeable improvement over those of [19] and [13.

4. Security assumption: Our scheme is proved to be secure (in the random oracle model) based on the worst-case hardness of approximating the Shortest Independent Vectors Problem, for general lattices of dimension $n$, to within a factor $\gamma=\widetilde{\mathcal{O}}\left(n^{1.5}\right)$. Surprisingly, this security assumption is as weak as those of state-of-the-art lattice-based standard signatures, such as [18, [14, and 29. This is a non-trivial feature, as group signatures are more elaborate primitive than standard signatures, one would expect to rely on a stronger security assumption. 
Overview of Our Techniques. The main building block of our VLR group signature scheme is an interactive protocol allowing a prover to convince the verifier that he is a certified group member (i.e., he possesses a valid secret signing key), and that he has not been revoked (i.e., his "revocation token" is not in the verifier's blacklist). The protocol is repeated many times to make the soundness error negligibly small, and then is converted to a signature scheme via Fiat-Shamir heuristic. Roughly speaking, in the random oracle model, the traceability and anonymity of the resulting group signature are based on the facts that the underlying protocol is a proof of knowledge, and it can be simulated.

We consider a group of $N=2^{\ell}$ users, where each user is identified by a string $d \in\{0,1\}^{\ell}$ denoting the binary representation of his index in the group. Let $n, m, \beta$, and $q \geq 2$ be integers (to be determined later). Our scheme operates within the structure of a Bonsai tree of hard random lattices 14, i.e., a matrix $\mathbf{A}=\left[\mathbf{A}_{0}\left|\mathbf{A}_{1}^{0}\right| \mathbf{A}_{1}^{1}|\ldots| \mathbf{A}_{\ell}^{0} \mid \mathbf{A}_{\ell}^{1}\right] \in \mathbb{Z}_{q}^{n \times(2 \ell+1) m}$, and a vector $\mathbf{u} \in \mathbb{Z}_{q}^{n}$. Initially, the group user with identity $d=d[1] \ldots d[\ell] \in\{0,1\}^{\ell}$ is issued a Bonsai signature of his identity, that is a small vector $\mathbf{z} \in \mathbb{Z}^{(\ell+1) m}$, such that $\|\mathbf{z}\|_{\infty} \leq \beta$ and $\mathbf{A}_{d} \cdot \mathbf{z}=\mathbf{u}$ mod $q$, where $\mathbf{A}_{d}=\left[\mathbf{A}_{0}\left|\mathbf{A}_{1}^{d[1]}\right| \ldots \mid \mathbf{A}_{\ell}^{d[\ell]}\right]$ - a subtree defined by $d$. In other words, vector $\mathbf{z}$ is a solution to the Inhomogeneous Small Integer Solution (ISIS) instance $\left(\mathbf{A}_{d}, \mathbf{u}\right)$. To prove that he is a certified group member without leaking $\mathbf{z}$, the user can perform a proof of knowledge (e.g., [32|28|27]) to convince the verifier that he knows such a vector $\mathbf{z}$ in zero-knowledge.

At this stage, one can obtain a secure identity-based identification scheme (as shown in (38), but it is insufficient for our purposes: to achieve anonymity, the group user also has to hide his identity $d$, and hence the matrix $\mathbf{A}_{d}$ should not be explicitly given. This raises an interesting question: If the verifier does not know $\mathbf{A}_{d}$, how could he be convinced that $\mathbf{A}_{d} \cdot \mathbf{z}=\mathbf{u} \bmod q$ ? To address this issue, we introduce the following extension: we add $\ell$ suitable zero-blocks of size $m$ to vector $\mathbf{z}$ to obtain an extended vector $\mathbf{x}=\left(\mathbf{x}_{0}\left\|\mathbf{x}_{1}^{0}\right\| \mathbf{x}_{1}^{1}\|\ldots\| \mathbf{x}_{\ell}^{0} \| \mathbf{x}_{\ell}^{1}\right) \in \mathbb{Z}^{(2 \ell+1) m}$, where the added zero-blocks are $\mathbf{x}_{1}^{1-d[1]}, \ldots, \mathbf{x}_{\ell}^{1-d[\ell]}$. We then have $\|\mathbf{x}\|_{\infty} \leq \beta$, and $\mathbf{A} \cdot \mathbf{x}=\mathbf{u} \bmod q$. Namely $\mathbf{x}$ is a solution to the ISIS instance given by the whole Bonsai tree, with an additional condition: for each $i=1, \ldots, \ell$, one of the two blocks $\mathbf{x}_{i}^{0}, \mathbf{x}_{i}^{1}$ must be zero, where the arrangement of the zero-blocks is determined by $d$. To prove in zero-knowledge the possession of such a vector $\mathbf{x}$, we adapt the 'Stern Extension' proof system from [27, where the user identity $d$ is hidden by a "one-time pad" technique. This technique is as follows. In each round of the protocol, the user samples a fresh uniformly random $e \in\{0,1\}^{\ell}$ and permutes the blocks of $\mathbf{x}$ to obtain the permuted vector $\mathbf{v}$, whose zero-blocks are arranged according to $d \oplus e$ (where $\oplus$ denotes the bit XOR operation). Depending on the verifier's challenge, the user later will either reveal $e$, or reveal $d \oplus e$ and show that $\mathbf{v}$ has the correct shape determined by $d \oplus e$. Since $d \oplus e$ is uniformly random over $\{0,1\}^{\ell}$, the user identity $d$ is completely hidden. As a result, the user can anonymously prove his group membership.

We now briefly review our revocation mechanism. For each group user's secret key $\mathbf{x}$, consider the first block $\mathbf{x}_{0}$ that corresponds to the "root" $\mathbf{A}_{0}$ of the Bonsai tree, and let his revocation token be $\mathbf{A}_{0} \cdot \mathbf{x}_{0} \bmod q \in \mathbb{Z}_{q}^{n}$. We choose suitable 
parameters, and sample $\mathbf{x}_{0}$ from a proper distribution, so that the token is statistically close to uniform over $\mathbb{Z}_{q}^{n}$. At a high level, our revocation mechanism works as follows. The user is asked to sample a uniformly random vector $\mathbf{r}_{0} \in \mathbb{Z}_{q}^{m}$, and to compute a commitment $\mathbf{c}_{0}$ using a (lattice-based) statistically hiding and computationally binding string commitment scheme COM, for which the value $\mathbf{A}_{0} \cdot \mathbf{r}_{0} \bmod q$ is part of the committed string. Depending on the verifier's challenge, the user will either reveal $\mathbf{r}_{0}$ or reveal $\mathbf{x}_{0}+\mathbf{r}_{0}$. In the former case, the verifier can check for honest computation of $\mathbf{c}_{0}$, while in the latter case, he can perform the revocation check using a list of tokens of revoked users $R L=\left\{\left\{\mathbf{u}_{i}\right\}_{i}\right\} \subset \mathbb{Z}_{q}^{n}$, as follows: For all $\mathbf{u}_{i} \in R L$, check that $\mathbf{c}_{0} \neq \operatorname{COM}\left(\mathbf{A}_{0}\right.$. $\left.\left(\mathbf{x}_{0}+\mathbf{r}_{0}\right)-\mathbf{u}_{i} \bmod q\right)$. Assuming that the user has been revoked, i.e., there exists $i$ such that $\mathbf{A}_{0} \cdot \mathbf{x}_{0} \bmod q=\mathbf{u}_{i}$. If he follows the protocol, then $\operatorname{COM}\left(\mathbf{A}_{0}\right.$. $\left.\left(\mathbf{x}_{0}+\mathbf{r}_{0}\right)-\mathbf{u}_{i} \bmod q\right)=\operatorname{COM}\left(\mathbf{A}_{0} \cdot \mathbf{r}_{0} \bmod q\right)=\mathbf{c}_{0}$, and thus, he gets rejected. If there is a false acceptance, then we can use it to break the computational binding property of COM. On the other hand, the probability of false rejection is negligibly small, since COM is statistically regular.

Putting everything together, we obtain a lattice-based VLR group signature that has several nice features, as mentioned earlier. In the process, we exploit the rich structure of the Bonsai tree [14], and the versatility of the "Stern Extension" proof system [27]. We also employ a special "one-time pad" technique, and a novel revocation mechanism.

\section{Preliminaries}

Notations. Vectors are denoted in bold lower-case letters and matrices in bold upper-case letters. We assume that all vectors are column vectors. The concatenation of vectors $\mathbf{x} \in \mathbb{R}^{m}$ and $\mathbf{y} \in \mathbb{R}^{k}$ is denoted by $(\mathbf{x} \| \mathbf{y})$. We denote the column concatenation of matrices $\mathbf{A} \in \mathbb{R}^{n \times m}$ and $\mathbf{B} \in \mathbb{R}^{n \times k}$ by $[\mathbf{A} \mid \mathbf{B}]$. Let $\mathbf{x}=\left(x_{i}\right)_{1 \leq i \leq n}$, we denote by Parse $\left(\mathbf{x}, i_{1}, i_{2}\right)$ the vector $\left(x_{i}\right)_{i_{1} \leq i \leq i_{2}}$ for $i_{1}, i_{2} \in[n]$. If $\mathrm{S}$ is a finite set, $y \stackrel{\$}{\leftarrow} \mathrm{S}$ means that $y$ is chosen uniformly at random from $\mathrm{S}$. If $D_{1}$ and $D_{2}$ are two distributions over the same countable support $S$, then their statistical distance is defined as $\Delta\left(D_{1}, D_{2}\right)=\frac{1}{2} \sum_{x \in S}\left|D_{1}(x)-D_{2}(x)\right|$. Two distributions are statistically close if their statistical distance is negligible.

\subsection{VLR Group Signature}

The presentation in this section follows [9]. A VLR group signature consists of 3 following algorithms:

- KeyGen $(n, N)$ : On input a security parameter $n$ and the number of group users $N$, this PPT algorithm outputs a group public key gpk, a vector of user secret keys gsk $=(\operatorname{gsk}[0], \operatorname{gsk}[1], \ldots, \operatorname{gsk}[N-1])$, and a vector of user revocation tokens $\operatorname{grt}=(\operatorname{grt}[0], \operatorname{grt}[1], \ldots, \operatorname{grt}[N-1])$.

- $\operatorname{Sign}($ gpk, gsk $[d], M)$ : On input gpk, a user secret key gsk $[d]$, and a message $M \in\{0,1\}^{*}$, this PPT algorithm outputs a signature $\Sigma$. 
- Verify (gpk, $R L, \Sigma, M)$ : On input gpk, a set of revocation tokens $R L \subseteq$ $\{\operatorname{grt}[0], \operatorname{grt}[1], \ldots, \operatorname{grt}[N-1]\}$, a signature $\Sigma$, and the message $M$, this algorithm outputs either Valid or Invalid. The output Valid indicates that $\Sigma$ is a valid signature on message $M$ under gpk, and the signer has not been revoked.

Remark 1. Any VLR group signature has an implicit tracing algorithm using grt as the tracing key. The tracing algorithm works as follows: on input a valid signature $\Sigma$ on a message $M$, it reveals the signer of $\Sigma$ by running Verify (gpk, $R L=$ $\operatorname{grt}[d], \Sigma, M)$, for $d=0,1, \ldots$, and outputting the first index $d^{*} \in\{0,1, \ldots, N-$ $1\}$ for which the verification algorithm returns Invalid. The tracing algorithm fails if and only if the given signature is properly verified for all $d$.

A secure VLR group signature scheme must satisfy the following 3 requirements:

1. Correctness: For all (gpk, gsk, grt) outputted by KeyGen, $M \in\{0,1\}^{*}$, and $d \in\{0,1, \ldots, N-1\}$ :

$$
\text { Verify }(\text { gpk, } R L, \operatorname{Sign}(\text { gpk, gsk }[d], M), M)=\text { Valid } \Leftrightarrow \operatorname{grt}[d] \notin R L \text {. }
$$

2. Selfless-anonymity: In the following selfless-anonymity game, the adversary's goal is to determine which of the two adaptively chosen keys generated a signature. He is not given access to either key.

3. Traceability: The adversary's goal in the traceability game is to forge a signature that cannot be traced to one of the users in his coalition using the implicit tracing algorithm above.

The formal definitions of the selfless-anonymity and traceability games can be found at [9, Sec. 2] and in the full version of the present paper [24].

\subsection{Some Cryptographic Tools from Lattices}

Lattices. Let $n, m$, and $q \geq 2$ be integers. For matrix $\mathbf{A} \in \mathbb{Z}_{q}^{n \times m}$, define the $m$-dimensional lattice: $\Lambda^{\perp}(\mathbf{A})=\left\{\mathbf{x} \in \mathbb{Z}^{m}: \mathbf{A} \cdot \mathbf{x}=\mathbf{0} \bmod q\right\} \subseteq \mathbb{Z}^{m}$. For any $\mathbf{u}$ in the image of $\mathbf{A}$, define the $\operatorname{coset} \Lambda_{\mathbf{u}}^{\perp}(\mathbf{A})=\left\{\mathbf{x} \in \mathbb{Z}^{m}: \mathbf{A} \cdot \mathbf{x}=\mathbf{u} \bmod q\right\}$. We recall the homogeneous and inhomogeneous Small Integer Solution problems (SIS and ISIS).

Definition 1. The $\operatorname{SIS}_{n, m, q, \beta}^{p}$ and $\operatorname{ISIS}_{n, m, q, \beta}^{p}$ problem in the $\ell_{p}$ norm with parameters $(n, m, q, \beta)$ are as follows: Given a uniformly random matrix $\mathbf{A} \in$ $\mathbb{Z}_{q}^{n \times m}$, and a uniformly random vector $\mathbf{u} \in \mathbb{Z}_{q}^{n}$,

- $\mathrm{SIS}_{n, m, q, \beta}^{p}$ asks to find a non-zero vector $\mathbf{x} \in \Lambda^{\perp}(\mathbf{A})$ such that $\|\mathbf{x}\|_{p} \leq \beta$.

- $\mathrm{ISIS}_{n, m, q, \beta}^{p}$ asks to find a vector $\mathbf{x} \in \Lambda_{\mathbf{u}}^{\perp}(\mathbf{A})$ such that $\|\mathbf{x}\|_{p} \leq \beta$.

The hardness of the SIS and ISIS problems is given by a worst-case to averagecase reduction from standard lattice problems, such as the Shortest Independent Vectors Problem (SIVP). 
Theorem 1 ([18]). For any $m, \beta=\operatorname{poly}(n)$, and for any $q \geq \beta \cdot \omega(\sqrt{n \log n})$, solving a random instance of the $\mathrm{SIS}_{n, m, q, \beta}^{2}$ or $\mathrm{ISIS}_{n, m, q, \beta}^{2}$ problem with nonnegligible probability is at least as hard as approximating the $\mathrm{SIVP}_{\gamma}^{2}$ problem on any lattice of dimension $n$ to within certain $\gamma=\beta \cdot \widetilde{O}(\sqrt{n})$ factors.

It then follows from the relationship between the $\ell_{2}$ and $\ell_{\infty}$ norms that the $\mathrm{SIS}_{n, m, q, \beta}^{\infty}$ and $\operatorname{ISIS}_{n, m, q, \beta}^{\infty}$ problems are at least as hard as $\operatorname{SIVP}_{\gamma}^{2}$ (in the $\ell_{2}$ norm) for some $\gamma=\beta \cdot \widetilde{O}(n)$.

Gaussians over Lattices. For any positive real $\sigma$, the $n$-dimensional Gaussian function is defined as: $\forall \mathbf{x} \in \mathbb{R}^{n}, \rho_{\sigma}(\mathbf{x})=\exp \left(-\pi\|\mathbf{x}\|^{2} / \sigma^{2}\right)$. For any $n$-dimensional lattice $\Lambda$, define the discrete Gaussian distribution over $\Lambda$ as: $\forall \mathbf{x} \in \Lambda, D_{\Lambda, \sigma}(\mathbf{x})=$ $\frac{\rho_{\sigma}(\mathbf{x})}{\rho_{\sigma}(\Lambda)}$. In the following lemma, we review several well-known facts about discrete Gaussian distribution:

Lemma 1 ([18] [36]). Let $n$ and $q \geq 2$ be integers. Let $m \geq 2 n \log q$, and $\sigma \geq \omega(\sqrt{\log m})$.

1. For all but a $2 q^{-n}$ fraction of all $\mathbf{A} \in \mathbb{Z}_{q}^{n \times m}$, for $\mathbf{x} \hookleftarrow D_{\mathbb{Z}^{m}, \sigma}$, the distribution of $\mathbf{u}=\mathbf{A} \cdot \mathbf{x} \bmod q$ is statistically close to uniform over $\mathbb{Z}_{q}^{n}$. Moreover, the conditional distribution of $\mathbf{x}$ given $\mathbf{u}$ is $D_{\Lambda_{\mathbf{u}}^{\perp}(\mathbf{A}), \sigma}$.

2. For $\beta=\lceil\sigma \cdot \log m\rceil$, and $\mathbf{x} \hookleftarrow D_{\mathbb{Z}^{m}, \sigma}, \operatorname{Pr}\left[\|\mathbf{x}\|_{\infty}>\beta\right]$ is negligible.

3. The min-entropy of $D_{\mathbb{Z}^{m}, \sigma}$ is at least $m-1$.

We now recall results about two fundamental tools: the trapdoor generation and the preimage sampling algorithms. The following algorithms are improvements of those in the literature [2/8/35].

Theorem 2 ([30]). Given integers $n \geq 1, q \geq 2$, and $m \geq 2 n \log q$. There is a PPT algorithm $\operatorname{Gen} \operatorname{Trap}(n, m, q)$ that outputs a matrix $\mathbf{A} \in \mathbb{Z}_{q}^{n \times m}$ and a trapdoor $\mathbf{R}_{\mathbf{A}}$, such that the distribution of $\mathbf{A}$ is negl $(n)$-far from uniform. Moreover, for any vector $\mathbf{u}$ in the image of $\mathbf{A}$ and $\sigma=\omega(\sqrt{n \log q \log n})$, there is a PPT algorithm SampleD $\left(\mathbf{R}_{\mathbf{A}}, \mathbf{A}, \mathbf{u}, \sigma\right)$ that outputs $\mathbf{x} \in \mathbb{Z}^{m}$ sampled from the distribution $D_{\mathbb{Z}^{m}, \sigma}$, conditioned on the event that $\mathbf{A} \cdot \mathbf{x}=\mathbf{u} \bmod q$.

The KTX String Commitment Scheme. Kawachi et al. 21 gave a string commitment scheme COM $:\{0,1\}^{*} \times\{0,1\}^{\bar{m} / 2} \rightarrow \mathbb{Z}_{q}^{n}$, such that:

- If $\bar{m}>2 n(1+\delta) \log q$ for $\delta>0$ constant, COM is statistically hiding.

- If the $\mathrm{SIS}_{n, \bar{m}, q, 1}^{\infty}$ problem is hard, then COM is computationally binding.

In this paper, we extensively use the KTX commitment scheme. For simplicity, we omit the randomness of the commitment. Also, we choose $\bar{m}$ sufficiently large, e.g., $\bar{m}=4 n \log q$, to make COM statistically hiding.

\section{Preparations}

In this section, we will describe the parameters and some specific constructions that will be used in our VLR group signature scheme. 


\subsection{Parameters}

Our group signature scheme involves 2 main parameters: a security parameter $n$ and a desired number of group users $N=2^{\ell} \in \operatorname{poly}(n)$. Given $n$, we fix the other scheme parameters as in Table 1.

Table 1. Parameters of our VLR group signature scheme. The sequence $\beta_{1}, \beta_{2}, \ldots, \beta_{p}$ satisfies $\sum_{j=1}^{p} \beta_{j}=\beta$, and every integer in the interval $[-\beta, \beta]$ can be efficiently expressed as a subset sum of elements in the set $\left\{ \pm \beta_{1}, \pm \beta_{2}, \ldots, \pm \beta_{p}\right\}$.

\begin{tabular}{|c|c|}
\hline Parameter & Value or Asymptotic bound \\
\hline Modulus $q$ & $\omega\left(n^{2} \log n\right)$ \\
\hline Dimension $m$ & $\geq 2 n \log q$ \\
\hline Gaussian parameter $\sigma$ & $\omega(\sqrt{n \log q \log n})$ \\
\hline Integer norm bound $\beta$ & $\lceil\sigma \cdot \log m\rceil$ \\
\hline Number of 'decompositions' $p$ & $\lfloor\log \beta\rfloor+1$ \\
\hline Sequence of integers \\
$\beta_{1}, \beta_{2}, \beta_{3}, \ldots, \beta_{p}$ & $\beta_{1}=\lceil\beta / 2\rceil ; \beta_{2}=\left\lceil\left(\beta-\beta_{1}\right) / 2\right\rceil$ \\
$\beta_{3}=\left\lceil\left(\beta-\beta_{1}-\beta_{2}\right) / 2\right\rceil ; \ldots ; \beta_{p}=1$ \\
\hline Number of protocol repetitions $t$ & $\omega(\log n)$ \\
\hline
\end{tabular}

\subsection{Some Specific Sets}

We now define some specific sets of vectors and permutations that will be extensively used throughout this work. First, we denote by $\mathrm{B}_{3 m}$ the set of all vectors in $\{-1,0,1\}^{3 m}$ having exactly $m$ coordinates $-1 ; m$ coordinates 0 ; and $m$ coordinates 1 . Given a binary string $d=d[1] \ldots d[\ell] \in\{0,1\}^{\ell}$, we define two sets:

- $\operatorname{Secret}_{\beta}(d)$ : The set of all $\mathbf{x}=\left(\mathbf{x}_{0}\left\|\mathbf{x}_{1}^{0}\right\| \mathbf{x}_{1}^{1}\|\ldots\| \mathbf{x}_{\ell}^{0} \| \mathbf{x}_{\ell}^{1}\right) \in \mathbb{Z}^{(2 \ell+1) m}$ consisting of $2 \ell+1$ blocks of size $m$, such that $\|\mathbf{x}\|_{\infty} \leq \beta$, and the following $\ell$ blocks are zero-blocks $\mathbf{0}^{m}: \mathbf{x}_{1}^{1-d[1]}, \ldots, \mathbf{x}_{\ell}^{1-d[\ell]}$.

- SecretExt $(d)$ : The set of all vectors $\mathbf{x}=\left(\mathbf{x}_{0}\left\|\mathbf{x}_{1}^{0}\right\| \mathbf{x}_{1}^{1}\|\ldots\| \mathbf{x}_{\ell}^{0} \| \mathbf{x}_{\ell}^{1}\right) \in$ $\{-1,0,1\}^{(2 \ell+1) 3 m}$ consisting of $2 \ell+1$ blocks of size $3 m$, such that the $\ell+1$ blocks $\mathbf{x}_{0}, \mathbf{x}_{1}^{d[1]}, \ldots, \mathbf{x}_{\ell}^{d[\ell]}$ are elements of $\mathrm{B}_{3 m}$, and the remaining $\ell$ blocks $\mathbf{x}_{1}^{1-d[1]}, \ldots, \mathbf{x}_{\ell}^{1-d[\ell]}$ are zero-blocks $\mathbf{0}^{3 m}$.

Given a vector $\mathbf{x}=\left(\mathbf{x}_{0}\left\|\mathbf{x}_{1}^{0}\right\| \mathbf{x}_{1}^{1}\|\ldots\| \mathbf{x}_{\ell}^{0} \| \mathbf{x}_{\ell}^{1}\right) \in \mathbb{Z}^{(2 \ell+1) 3 m}$ consisting of $2 \ell+1$ blocks of size $3 m$, we define two sets of permutations of $\mathbf{x}$ :

- The set $\mathcal{S}$ of all permutations that keep the arrangement of the blocks: If $\pi \in \mathcal{S}$, then $\pi(\mathbf{x})=\left(\tau_{0}\left(\mathbf{x}_{0}\right)\left\|\tau_{1}^{0}\left(\mathbf{x}_{1}^{0}\right)\right\| \tau_{1}^{1}\left(\mathbf{x}_{1}^{1}\right)\|\ldots\| \tau_{\ell}^{0}\left(\mathbf{x}_{\ell}^{0}\right) \| \tau_{\ell}^{1}\left(\mathbf{x}_{\ell}^{1}\right)\right)$, where $\tau_{0}, \tau_{1}^{0}, \tau_{1}^{1}, \ldots, \tau_{\ell}^{0}, \tau_{\ell}^{1}$ are certain permutations of $3 m$ elements.

- The set $\mathcal{T}=\left\{T_{e} \mid e \in\{0,1\}^{\ell}\right\}$, where for $e=e[1] \ldots e[\ell], T_{e} \in \mathcal{T}$ rearranges the blocks as: $T_{e}(\mathbf{x})=\left(\mathbf{x}_{0}\left\|\mathbf{x}_{1}^{e[1]}\right\| \mathbf{x}_{1}^{1-e[1]}\|\ldots\| \mathbf{x}_{\ell}^{e[\ell]} \| \mathbf{x}_{\ell}^{1-e[\ell]}\right)$. 
In particular, given $d, e \in\{0,1\}^{\ell}, \pi \in \mathcal{S}$, and $\mathbf{x} \in \mathbb{Z}^{(2 \ell+1) 3 m}$, it can be checked that:

$$
\mathbf{x} \in \operatorname{SecretExt}(d) \Leftrightarrow \pi(\mathbf{x}) \in \operatorname{SecretExt}(d) \Leftrightarrow T_{e} \circ \pi(\mathbf{x}) \in \operatorname{SecretExt}(d \oplus e) .
$$

\subsection{The Decomposition - Extension Technique}

Ling et al. 27] proposed a Stern-type zero-knowledge proof of knowledge for the $\operatorname{ISIS}_{n, m, q, \beta}^{\infty}$ problem, which relies on a Decomposition-Extension framework. Adapting their technique, we construct the following procedures:

Elementary Decomposition. On input a vector $\mathbf{v}=\left(v_{1}, v_{2}, \ldots, v_{m}\right) \in \mathbb{Z}^{m}$ such that $\|\mathbf{v}\|_{\infty} \leq \beta$, the procedure EleDec outputs $p=\lfloor\log \beta\rfloor+1$ vectors $\widetilde{\mathbf{w}}_{1}, \ldots, \widetilde{\mathbf{w}}_{p} \in\{-1,0,1\}^{m}$, such that $\sum_{j=1}^{p} \beta_{j} \cdot \widetilde{\mathbf{w}}_{j}=\mathbf{v}$. This procedure works as follows:

1. For each $i \in[m]$, express $v_{i}$ as $v_{i}=\beta_{1} \cdot v_{i, 1}+\beta_{2} \cdot v_{i, 2}+\ldots+\beta_{p} \cdot v_{i, p}$, where $\forall j \in[p]: v_{i, j} \in\{-1,0,1\}$. It was noted in [27] that for $\beta_{1}, \beta_{2}, \ldots, \beta_{p}$ given in Table 1, this step can easily be done.

2. For each $j \in[p]$, let $\widetilde{\mathbf{w}}_{j}:=\left(v_{1, j}, v_{2, j}, \ldots, v_{m, j}\right) \in\{-1,0,1\}^{m}$. Output $\widetilde{\mathbf{w}}_{1}, \ldots, \widetilde{\mathbf{w}}_{p}$.

Elementary Extension. On input a vector $\widetilde{\mathbf{w}} \in\{-1,0,1\}^{m}$, EleExt extends $\widetilde{\mathbf{w}}$ to a vector $\mathbf{w} \in \mathrm{B}_{3 m}$. This procedure works as follows:

1. Let $\lambda^{(-1)}, \lambda^{(0)}$ and $\lambda^{(1)}$ be the numbers of coordinates of $\widetilde{\mathbf{w}}$ that equal to $-1,0$, and 1 respectively.

2. Pick a random vector $\widehat{\mathbf{w}} \in\{-1,0,1\}^{2 m}$ that has exactly $\left(m-\lambda^{(-1)}\right)$ coordinates $-1,\left(m-\lambda^{(0)}\right)$ coordinates 0 , and $\left(m-\lambda^{(1)}\right)$ coordinates 1 . Output $\mathbf{w}=(\widetilde{\mathbf{w}} \| \widehat{\mathbf{w}}) \in \mathrm{B}_{3 m}$.

Witness Decomposition and Extensions. On input $\mathbf{x} \in \operatorname{Secret}_{\beta}(d)$ for some $d=d[1] \ldots d[\ell] \in\{0,1\}^{\ell}$, the procedure WitnessDE outputs $p$ vectors $\mathbf{z}_{1}, \ldots \mathbf{z}_{p} \in$ SecretExt $(d)$. This procedure works as follows:

1. Write $\mathbf{x}$ as the concatenation of $2 \ell+1$ blocks of size $m$, namely: $\mathbf{x}=$ $\left(\mathbf{x}_{0}\left\|\mathbf{x}_{1}^{0}\right\| \mathbf{x}_{1}^{1}\|\ldots\| \mathbf{x}_{\ell}^{0} \| \mathbf{x}_{\ell}^{1}\right)$.

2. Run EleDec on each of the $\ell+1$ blocks $\mathbf{x}_{0}, \mathbf{x}_{1}^{d[1]}, \ldots, \mathbf{x}_{\ell}^{d[\ell]}$ to obtained $(\ell+1) p$ decomposed vectors. Then run EleExt on each of the decomposed vectors to obtain $(\ell+1) p$ vectors in $\mathrm{B}_{3 m}$, denoted respectively by $\left\{\mathbf{w}_{0, j}\right\}_{j=1}^{p},\left\{\mathbf{w}_{1, j}^{d[1]}\right\}_{j=1}^{p}, \ldots,\left\{\mathbf{w}_{\ell, j}^{d[\ell]}\right\}_{j=1}^{p}$.

3. Create $\ell p$ zero-vectors of dimension $3 m$, and denote them by: $\left\{\mathbf{w}_{1, j}^{1-d[1]}\right\}_{j=1}^{p}, \ldots,\left\{\mathbf{w}_{\ell, j}^{1-d[\ell]}\right\}_{j=1}^{p}$.

4. For each $j \in[p]$, let $\mathbf{z}_{j}=\left(\mathbf{w}_{0, j}\left\|\mathbf{w}_{1, j}^{0}\right\| \mathbf{w}_{1, j}^{1}\|\ldots\| \mathbf{w}_{\ell, j}^{0} \| \mathbf{w}_{\ell, j}^{1}\right)$. Output $\mathbf{z}_{1}, \ldots, \mathbf{z}_{p}$ $\in \operatorname{SecretExt}(d)$.

Matrix Extension. On input $\mathbf{A} \in \mathbb{Z}_{q}^{n \times(2 \ell+1) m}$, the following procedure MatrixExt outputs $\mathbf{A}^{*} \in \mathbb{Z}_{q}^{n \times(2 \ell+1) 3 m}$ : 
1. Write $\mathbf{A}$ as the concatenation of $2 \ell+1$ component-matrices in $\mathbb{Z}_{q}^{n \times m}$.

2. Append $2 m$ zero-columns to each of the component-matrices, then output the extended matrix $\mathbf{A}^{*}$.

In particular, let $\left\{\mathbf{z}_{j}\right\}_{j=1}^{p} \leftarrow$ WitnessDE( $(\mathbf{x})$ and $\mathbf{A}^{*} \leftarrow \operatorname{MatrixExt}(\mathbf{A})$ then we have $\mathbf{A} \cdot \mathbf{x}=\mathbf{A}^{*} \cdot\left(\sum_{j=1}^{p} \beta_{j} \cdot \mathbf{z}_{j}\right)$. We illustrate our technique in Figure 1 .
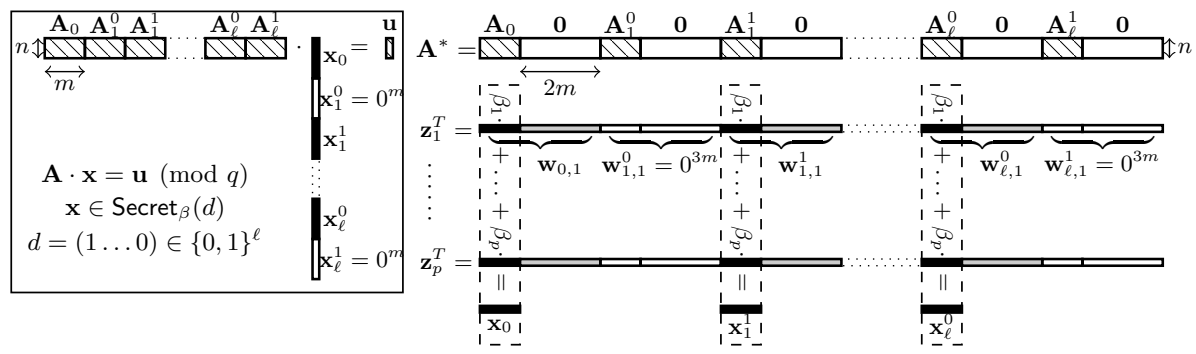

Fig. 1. An illustration of our Decomposition-Extension technique, where the first bit of $d$ is 1 and its last bit is 0 . We denote by an element of $\mathrm{B}_{3 m}$. After performing Decomposition-Extension, one has that $\mathbf{z}_{j} \in \operatorname{SecretExt}(d)$ for all $j \in[p]$, and $\mathbf{A}^{*} \cdot\left(\sum_{j=1}^{p} \beta_{j} \cdot \mathbf{z}_{j}\right)=\mathbf{A} \cdot \mathbf{x}=\mathbf{u} \bmod q$.

Therefore, in the protocol in Section 4, to prove that $\mathbf{x} \in \operatorname{Secret}_{\beta}(d)$ for some $d \in\{0,1\}^{\ell}$, and $\mathbf{A} \cdot \mathbf{x}=\mathbf{u} \bmod q$, one can instead prove that:

$$
\left\{\begin{array}{l}
\mathbf{A}^{*} \cdot\left(\sum_{j=1}^{p} \beta_{j} \cdot \mathbf{z}_{j}\right)=\mathbf{u} \bmod q \\
\forall j \in[p], \pi \in \mathcal{S}, e \in\{0,1\}^{\ell}: T_{e} \circ \pi\left(\mathbf{z}_{j}\right) \in \operatorname{SecretExt}(d \oplus e),
\end{array}\right.
$$

where the second relation follows from the fact that $\mathbf{z}_{j} \in \operatorname{SecretExt}(d)$ for all $j \in[p]$, and from (11).

\section{The Underlying Interactive Protocol}

We recall that the main building block of our VLR group signature scheme is an interactive protocol that allows the prover to convince the verifier that he is a certified group member (i.e., he has a valid secret key), and that he has not been revoked (i.e., his revocation token is not in the verifier's list $R L$ ). In Section 5 , the protocol is repeated $t=\omega(\log n)$ times to make the soundness error negligibly small, and then is transform to a signature scheme via Fiat-Shamir heuristic. The interactive protocol is summarized as follows:

- The public parameters are $\mathbf{A}=\left[\mathbf{A}_{0}\left|\mathbf{A}_{1}^{0}\right| \mathbf{A}_{1}^{1}|\ldots| \mathbf{A}_{\ell}^{0} \mid \mathbf{A}_{\ell}^{1}\right] \in \mathbb{Z}_{q}^{n \times(2 \ell+1) m}$ and $\mathbf{u} \in \mathbb{Z}_{q}^{n}$. 
- The prover's witness is a $\mathbf{x}=\left(\mathbf{x}_{0}\left\|\mathbf{x}_{1}^{0}\right\| \mathbf{x}_{1}^{1}\|\ldots\| \mathbf{x}_{\ell}^{0} \| \mathbf{x}_{\ell}^{1}\right) \in \operatorname{Secret}_{\beta}(d)$ for some $d \in\{0,1\}^{\ell}$. The verifier's additional input is a set $R L=\left\{\left\{\mathbf{u}_{i}\right\}_{i}\right\} \subset \mathbb{Z}_{q}^{n}$, whose cardinality is at most $N-1$.

- The prover's goal is to convince the verifier in that:

1. $\mathbf{A} \cdot \mathbf{x}=\mathbf{u} \bmod q$ and $\mathbf{x} \in \operatorname{Secret}_{\beta}(d)$, while keeping $d$ secret.

2. $\mathbf{A}_{0} \cdot \mathbf{x}_{0} \bmod q \notin R L$.

\subsection{Description of the Protocol}

Let COM be the KTX commitment scheme [21]. Let $\mathbf{A}^{*} \leftarrow \operatorname{MatrixExt}(\mathbf{A})$. Prior to the interaction, the prover applies the Decomposition-Extension technique on his witness: Let $\mathbf{z}_{1}, \ldots, \mathbf{z}_{p} \leftarrow$ WitnessDE( $(\mathbf{x})$. The protocol follows Stern's approach for three-pass zero-knowledge identification schemes [40, for which we employ an additional commitment $\mathbf{c}_{0}$ to enable the revocation mechanism. The details are as follows:

1. Commitment: The prover samples a string $e \stackrel{\$}{\leftarrow}\{0,1\}^{\ell}, p$ permutations $\pi_{1}, \ldots, \pi_{p} \stackrel{\$}{\leftarrow} \mathcal{S}$, and $p$ vectors $\mathbf{r}_{1}, \ldots, \mathbf{r}_{p} \stackrel{\$}{\leftarrow} \mathbb{Z}_{q}^{(2 \ell+1) \cdot 3 m}$. For each $j \in[p]$, let $\mathbf{r}_{j, 0}=\operatorname{Parse}\left(\mathbf{r}_{j}, 1, m\right)$. Then it sends the commitment CMT $=\left(\mathbf{c}_{0}, \mathbf{c}_{1}, \mathbf{c}_{2}, \mathbf{c}_{3}\right)$ $\in\left(\mathbb{Z}_{q}^{n}\right)^{4}$ to the verifier, where

$$
\left\{\begin{array}{l}
\mathbf{c}_{0}=\operatorname{CoM}\left(e,\left\{\pi_{j}\right\}_{j=1}^{p}, \mathbf{A}_{0} \cdot\left(\sum_{j=1}^{p} \beta_{j} \cdot \mathbf{r}_{j, 0}\right) \bmod q\right) \\
\mathbf{c}_{1}=\operatorname{CoM}\left(e,\left\{\pi_{j}\right\}_{j=1}^{p}, \mathbf{A}^{*} \cdot\left(\sum_{j=1}^{p} \beta_{j} \cdot \mathbf{r}_{j}\right) \bmod q\right) \\
\mathbf{c}_{2}=\operatorname{CoM}\left(\left\{\mathrm{T}_{e} \circ \pi_{j}\left(\mathbf{r}_{j}\right)\right\}_{j=1}^{p}\right) \\
\mathbf{c}_{3}=\operatorname{CoM}\left(\left\{\mathrm{T}_{e} \circ \pi_{j}\left(\mathbf{z}_{j}+\mathbf{r}_{j}\right)\right\}_{j=1}^{p}\right) .
\end{array}\right.
$$

2. Challenge: The verifier sends $C h \stackrel{\$}{\leftarrow}\{1,2,3\}$ to the prover.

3. Response: Depending on the challenge, the prover computes the response RSP differently:

- Case $C h=1: \forall j \in[p]$, let $\mathbf{v}_{j}=\mathrm{T}_{e} \circ \pi_{j}\left(\mathbf{z}_{j}\right), \mathbf{w}_{j}=\mathrm{T}_{e} \circ \pi_{j}\left(\mathbf{r}_{j}\right), d_{1}=d \oplus e$, and set:

$$
\mathrm{RSP}=\left(d_{1},\left\{\mathbf{v}_{j}\right\}_{j=1}^{p},\left\{\mathbf{w}_{j}\right\}_{j=1}^{p}\right) \text {. }
$$

- Case $C h=2: \forall j \in[p]$, let $\phi_{j}=\pi_{j}, \mathbf{s}_{j}=\mathbf{z}_{j}+\mathbf{r}_{j}, d_{2}=e$, and set:

$$
\mathrm{RSP}=\left(d_{2},\left\{\phi_{j}\right\}_{j=1}^{p},\left\{\mathbf{s}_{j}\right\}_{j=1}^{p}\right) \text {. }
$$

- Case $C h=3: \forall j \in[p]$, let $\psi_{j}=\pi_{j}, \mathbf{h}_{j}=\mathbf{r}_{j}, d_{3}=e$, and set:

$$
\mathrm{RSP}=\left(d_{3},\left\{\psi_{j}\right\}_{j=1}^{p},\left\{\mathbf{h}_{j}\right\}_{j=1}^{p}\right) .
$$

Verification: Receiving RSP, the verifier proceeds as follows:

- Case $C h=1$ : Parse RSP as in (3). Check that $\forall j \in[p]: \mathbf{v}_{j} \in \operatorname{SecretExt}\left(d_{1}\right)$, and that:

$$
\mathbf{c}_{2}=\operatorname{CoM}\left(\left\{\mathbf{w}_{j}\right\}_{j=1}^{p}\right) \text { and } \mathbf{c}_{3}=\operatorname{CoM}\left(\left\{\mathbf{v}_{j}+\mathbf{w}_{j}\right\}_{j=1}^{p}\right)
$$


- Case $C h=2$ : Parse RSP as in (44). $\forall j \in[p]$, let $\mathbf{s}_{j, 0}=\operatorname{Parse}\left(\mathbf{s}_{j}, 1, m\right)$. Check that:

$$
\left\{\begin{array}{l}
\forall \mathbf{u}_{i} \in R L: \mathbf{c}_{0} \neq \operatorname{COM}\left(d_{2},\left\{\phi_{j}\right\}_{j=1}^{p}, \mathbf{A}_{0} \cdot\left(\sum_{j=1}^{p} \beta_{j} \cdot \mathbf{s}_{j, 0}\right)-\mathbf{u}_{i} \bmod q\right) \\
\mathbf{c}_{1}=\operatorname{COM}\left(d_{2},\left\{\phi_{j}\right\}_{j=1}^{p}, \mathbf{A}^{*} \cdot\left(\sum_{j=1}^{p} \beta_{j} \cdot \mathbf{s}_{j}\right)-\mathbf{u} \bmod q\right) \\
\mathbf{c}_{3}=\operatorname{CoM}\left(\left\{\mathrm{T}_{d_{2}} \circ \phi_{j}\left(\mathbf{s}_{j}\right)\right\}_{j=1}^{p}\right) .
\end{array}\right.
$$

- Case $C h=3$ : Parse RSP as in (5). $\forall j \in[p]$, let $\mathbf{h}_{j, 0}=\operatorname{Parse}\left(\mathbf{h}_{j}, 1, m\right)$. Check that:

$$
\left\{\begin{array}{l}
\mathbf{c}_{0}=\operatorname{COM}\left(d_{3},\left\{\psi_{j}\right\}_{j=1}^{p}, \mathbf{A}_{0} \cdot\left(\sum_{j=1}^{p} \beta_{j} \cdot \mathbf{h}_{j, 0}\right) \bmod q\right) \\
\mathbf{c}_{1}=\operatorname{CoM}\left(d_{3},\left\{\psi_{j}\right\}_{j=1}^{p}, \mathbf{A}^{*} \cdot\left(\sum_{j=1}^{p} \beta_{j} \cdot \mathbf{h}_{j}\right) \bmod q\right) \\
\mathbf{c}_{2}=\operatorname{CoM}\left(\left\{\mathrm{T}_{d_{3}} \circ \psi_{j}\left(\mathbf{h}_{j}\right)\right\}_{j=1}^{p}\right) .
\end{array}\right.
$$

The verifier outputs Valid if and only if all the conditions hold. Otherwise, he outputs Invalid.

\subsection{Witness Extraction}

The following lemma says that in our protocol, one can extract a satisfying witness under specific conditions. The proof of the lemma is given in the full version [24, Appendix A].

Lemma 2. Assume that for a given commitment CMT, there exist 3 valid responses $\mathrm{RSP}^{(1)}, \mathrm{RSP}^{(2)}$, and $\mathrm{RSP}^{(3)}$ corresponding to all 3 possible values of the challenge Ch. If COM is a computationally binding commitment scheme, then one can efficiently extract a vector $\mathbf{y}$ such that $\mathbf{y}=\left(\mathbf{y}_{0}\left\|\mathbf{y}_{1}^{0}\right\| \mathbf{y}_{1}^{1}\|\ldots\| \mathbf{y}_{\ell}^{0} \| \mathbf{y}_{\ell}^{1}\right) \in$ $\mathbb{Z}^{(2 \ell+1) m}$ satisfying $\mathbf{A} \cdot \mathbf{y}=\mathbf{u} \bmod q, \mathbf{y} \in \operatorname{Secret}_{\beta}(d)$ for some $d \in\{0,1\}^{\ell}$, and $\mathbf{A}_{0} \cdot \mathbf{y}_{0} \bmod q \notin R L$.

\section{The VLR Group Signature Scheme}

In this Section we will describe and analyze our lattice-based VLR group signature scheme. The scheme uses the protocol in Section 4 as its building block.

\subsection{Description of the Scheme}

Keys Generation. The algorithm $\operatorname{KeyGen}(n, N)$, works as follows:

1. Run GenTrap $(n, m, q)$ to get $\mathbf{A}_{0} \in \mathbb{Z}_{q}^{n \times m}$ and trapdoor $\mathbf{R}$.

2. Sample $\mathbf{u} \stackrel{\$}{\leftarrow} \mathbb{Z}_{q}^{n}$, and $\mathbf{A}_{i}^{b} \stackrel{\$}{\leftarrow} \mathbb{Z}_{q}^{n \times m}$ for all $b \in\{0,1\}$ and $i \in[\ell]$. Then define the matrix $\mathbf{A}=\left[\mathbf{A}_{0}\left|\mathbf{A}_{1}^{0}\right| \mathbf{A}_{1}^{1}|\ldots| \mathbf{A}_{\ell}^{0} \mid \mathbf{A}_{\ell}^{1}\right] \in \mathbb{Z}_{q}^{n \times(2 \ell+1) m}$.

3. For group user with index $d \in\{0,1, \ldots, N-1\}$, let $d[1] \ldots d[\ell] \in\{0,1\}^{\ell}$ denote the binary representation of $d$, and do the following: 
(a) Sample vectors $\mathbf{x}_{1}^{d[1]}, \ldots, \mathbf{x}_{\ell}^{d[\ell]} \hookleftarrow D_{\mathbb{Z}^{m}, \sigma}$. Compute $\mathbf{z}=\sum_{i=1}^{\ell} \mathbf{A}_{i}^{d[i]}$. $\mathbf{x}_{i}^{d[i]} \bmod q$, and sample $\mathbf{x}_{0} \in \mathbb{Z}^{m}$ with $\mathbf{x}_{0} \hookleftarrow \operatorname{SampleD}\left(\mathbf{R}, \mathbf{A}_{0}, \mathbf{u}-\right.$ $\mathbf{z}, \sigma)$. Let $\mathbf{x}_{1}^{1-d[1]}, \ldots, \mathbf{x}_{\ell}^{1-d[\ell]}$ be zero-vectors $\mathbf{0}^{m}$, and define $\mathbf{x}^{(d)}=$ $\left(\mathbf{x}_{0}\left\|\mathbf{x}_{1}^{0}\right\| \mathbf{x}_{1}^{1}\|\ldots\| \mathbf{x}_{\ell}^{0} \| \mathbf{x}_{\ell}^{1}\right) \in \mathbb{Z}^{(2 \ell+1) m}$. If $\left\|\mathbf{x}^{(d)}\right\|_{\infty} \leq \beta$ then go to step (3b); else, repeat step (3a).

(b) Let the user secret key be gsk $[d]=\mathbf{x}^{(d)}$, and the revocation token be $\operatorname{grt}[d]=\mathbf{A}_{0} \cdot \mathbf{x}_{0} \in \mathbb{Z}_{q}^{n}$.

4. The algorithm outputs (gpk, gsk, grt), where gpk $=(\mathbf{A}, \mathbf{u})$; gsk $=$ $(\operatorname{gsk}[0], \operatorname{gsk}[1], \ldots, \operatorname{gsk}[N-1]) ; \operatorname{grt}=(\operatorname{grt}[0], \operatorname{grt}[1], \ldots, \operatorname{grt}[N-1])$.

Remark 2. We have some observations on the behaviour of the above key generation algorithm:

- By Theorem 2, the distribution of $\mathbf{A}_{0}$ generated by $\operatorname{GenTrap}(n, m, q)$ is statistically close to uniform over $\mathbb{Z}_{q}^{n \times m}$. Thus, the distribution of gpk output by $\operatorname{KeyGen}(n, N)$ is statistically close to uniform over $\mathbb{Z}_{q}^{n \times(2 \ell+1) m} \times \mathbb{Z}_{q}^{n}$. We note that the pair $(\mathbf{A}, \mathbf{u})$ resembles the Bonsai tree structure 14], where $\mathbf{A}_{0}$ is the "root" of the tree.

- In Step (3a), each coordinate of vector $\mathbf{x}^{(d)}$ is either 0 or distributed according to the distribution $D_{\mathbb{Z}, \sigma}$ (see Theorem 2 regarding the output distribution of algorithm SampleD). By setting $\beta=\lceil\sigma \cdot \log m\rceil$, we ensure that $\left\|\mathbf{x}^{(d)}\right\|_{\infty} \leq \beta$ with overwhelming probability (see Lemma11). Thus, the event that Step (3a) needs to be repeated only occurs with negligible probability.

- The secret key $\mathbf{x}^{(d)}$ of group user with index $d$ satisfies $\mathbf{A} \cdot \mathbf{x}^{(d)}=\mathbf{u} \bmod q$, and $\mathbf{x}^{(d)} \in \operatorname{Secret}_{\beta}(d)$.

- By Lemma 1 the distribution of each user revocation token $\operatorname{grt}[d]$ is statistically close to uniform over $\mathbb{Z}_{q}^{n}$. The trivial requirement is that the revocation tokens of two different group users must be different. In the very rare event of conflict (i.e., there exist $d_{1}, d_{2} \in\{0, \ldots, N-1\}$ such that $d_{2}>d_{1}$ and $\left.\operatorname{grt}\left[d_{1}\right]=\operatorname{grt}\left[d_{2}\right]\right)$, the algorithm simply re-samples the key and token for user with index $d_{2}$.

Signing Algorithm. Let $\mathcal{H}:\{0,1\}^{*} \rightarrow\{1,2,3\}^{t}$ be a hash function, modelled as a random oracle. Given gpk $=(\mathbf{A}, \mathbf{u})$, to sign a message $M \in\{0,1\}^{*}$ using the secret key gsk $[d]=\mathbf{x} \in \operatorname{Secret}_{\beta}(d)$, the user runs the randomized algorithm $\operatorname{Sign}(\mathrm{gpk}, \mathrm{gsk}[d], M)$, which is as follow:

1. Generate a proof that the user is a certified group members and that he has not been revoked. This is done by repeating $t=\omega(\log n)$ times the basic protocol from Section 4 with public parameter $(\mathbf{A}, \mathbf{u})$ and prover's witness $\mathbf{x}$, and then making it non-interactive with the Fiat-Shamir heuristic as a triple $\left(\left\{\mathrm{CMT}^{(k)}\right\}_{k=1}^{t}, \mathrm{CH},\left\{\mathrm{RSP}^{(k)}\right\}_{k=1}^{t}\right)$, where $\mathrm{CH}=\left(\left\{C h^{(k)}\right\}_{k=1}^{t}\right)=$ $\mathcal{H}\left(M,\left\{\mathrm{CMT}^{(k)}\right\}_{k=1}^{t}\right) \in\{1,2,3\}^{t}$.

2. Output the group signature:

$$
\Sigma=\left(M,\left\{\mathrm{CMT}^{(k)}\right\}_{k=1}^{t},\left\{C h^{(k)}\right\}_{k=1}^{t},\left\{\mathrm{RSP}^{(k)}\right\}_{k=1}^{t}\right) .
$$


Verification Algorithm. On input gpk $=(\mathbf{A}, \mathbf{u})$, a set of tokens $R L=$ $\left\{\left\{\mathbf{u}_{i}\right\}_{i}\right\} \subset \mathbb{Z}_{q}^{n}$ whose cardinality is at most $N-1$, a message $M \in\{0,1\}^{*}$, and a purported group signature $\Sigma$ on $M$, the verifier runs the deterministic algorithm Verify (gpk, $R L, \Sigma, M)$, which performs the following steps:

1. Parse the signature $\Sigma$ as in (6).

2. Check if $\left(C h^{(1)}, \ldots, C h^{(t)}\right)=\mathcal{H}\left(M, \mathrm{CMT}^{(1)}, \ldots, \mathrm{CMT}^{(t)}\right)$.

3. For $k=1$ to $t$, run the verification of the protocol from Section 4 to check the validity of $\mathrm{RSP}^{(k)}$ with respect to $\mathrm{CMT}^{(k)}$ and $C h^{(k)}$. If any of the conditions does not hold, then output Invalid and terminate.

4. Output Valid.

\subsection{Analysis of the Scheme}

We now will analyze the efficiency and security properties of the VLR group signature described in Section 5.1 .

Efficiency. The parameters in Table 1 are set so that all of the algorithms in Section 5.1 can be implemented in polynomial time. Asymptotically, the group public key has bit-size $\ell \cdot \widetilde{\mathcal{O}}\left(n^{2}\right)=\log N \cdot \widetilde{\mathcal{O}}\left(n^{2}\right)$, while the group signatures have bit-size $\ell \cdot \widetilde{\mathcal{O}}(n)=\log N \cdot \widetilde{\mathcal{O}}(n)$. The revocation check, i.e., the check against $\mathbf{c}_{0}^{(k)}$ in the case $C h^{(k)}=2$, runs in linear time in the number of revoked users, as it seems unavoidable for secure VLR group signature schemes.

Security. The correctness, selfless-anonymity, and traceability of our VLR group signature scheme are stated in theorems 3, 4, and 5, respectively. The proofs of these theorems are provided in the full version of the paper [24].

Theorem 3. The VLR group signature scheme is correct with overwhelming probability.

In the random oracle model, our scheme is selfless-anonymous.

Theorem 4. If COM is a statistically hiding string commitment scheme, then the VLR group signature scheme in Section 5.1] is selfless-anonymous in the random oracle model.

Finally, in the random oracle model, our VLR group signature scheme is traceable if the $\mathrm{SIS}_{n,(\ell+1) \cdot m, q, 2 \beta}^{\infty}$ problem is hard.

Theorem 5. If there is a traceability adversary $\mathcal{A}$ with success probability $\epsilon$ and running time $T$, then there is an algorithm $\mathcal{F}$ that solves the $\operatorname{SIS}_{n,(\ell+1) \cdot m, q, 2 \beta}^{\infty}$ problem with success probability $\epsilon^{\prime}>\left(1-(7 / 9)^{t}\right) \cdot \frac{1}{2 N}$, and running time $T^{\prime}=$ $32 \cdot T \cdot q_{\mathcal{H}} /\left(\epsilon-3^{-t}\right)+\operatorname{poly}(n, N)$, where $q_{\mathcal{H}}$ is the number of queries to the random oracle $\mathcal{H}:\{0,1\}^{*} \rightarrow\{1,2,3\}^{t}$. 
The results of Theorem 1 and Theorem 5 imply that the traceability of our scheme in the random oracle model can be based on the worst-case hardness of the SIVP $_{\gamma}^{2}$ problem, with $\gamma=2 \beta \cdot \widetilde{\mathcal{O}}(n)=\widetilde{\mathcal{O}}\left(n^{1.5}\right)$.

Acknowledgements. The authors would like to thank D. Stehlé, B. Libert, R. Bhattacharyya, J. Chen, and the anonymous reviewers for their helpful comments. The research is supported in part by the Singapore Ministry of Education under Research Grant MOE2013-T2-1-041. Adeline Langlois is supported in part by ERC Starting Grant ERC-2013-StG-335086-LATTAC.

\section{References}

1. Ajtai, M.: Generating Hard Instances of Lattice Problems (Extended Abstract). In: STOC, pp. 99-108. ACM (1996)

2. Ajtai, M.: Generating Hard Instances of the Short Basis Problem. In: Wiedermann, J., Van Emde Boas, P., Nielsen, M. (eds.) ICALP 1999. LNCS, vol. 1644, pp. 1-9. Springer, Heidelberg (1999)

3. Alwen, J., Peikert, C.: Generating Shorter Bases for Hard Random Lattices. Theory Comput. Syst. 48(3), 535-553 (2011)

4. Ateniese, G., Camenisch, J., Joye, M., Tsudik, G.: A Practical and Provably Secure Coalition-Resistant Group Signature Scheme. In: Bellare, M. (ed.) CRYPTO 2000. LNCS, vol. 1880, pp. 255-270. Springer, Heidelberg (2000)

5. Bellare, M., Micciancio, D., Warinschi, B.: Foundations of Group Signatures: Formal Definitions, Simplified Requirements, and a Construction Based on General Assumptions. In: Biham, E. (ed.) EUROCRYPT 2003. LNCS, vol. 2656, pp. 614-629. Springer, Heidelberg (2003)

6. Bellare, M., Shi, H., Zhang, C.: Foundations of Group Signatures: The Case of Dynamic Groups. In: Menezes, A. (ed.) CT-RSA 2005. LNCS, vol. 3376, pp. 136-153. Springer, Heidelberg (2005)

7. Bichsel, P., Camenisch, J., Neven, G., Smart, N.P., Warinschi, B.: Get Shorty via Group Signatures without Encryption. In: Garay, J.A., De Prisco, R. (eds.) SCN 2010. LNCS, vol. 6280, pp. 381-398. Springer, Heidelberg (2010)

8. Boneh, D., Boyen, X., Shacham, H.: Short Group Signatures. In: Franklin, M. (ed.) CRYPTO 2004. LNCS, vol. 3152, pp. 41-55. Springer, Heidelberg (2004)

9. Boneh, D., Shacham, H.: Group Signatures with Verifier-local Revocation. In: ACM-CCS, pp. 168-177. ACM (2004)

10. Brickell, E.: An Efficient Protocol for Anonymously Providing Assurance of the Container of the Private Key. Submitted to the Trusted Comp. Group (April 2003)

11. Camenisch, J., Groth, J.: Group Signatures: Better Efficiency and New Theoretical Aspects. In: Blundo, C., Cimato, S. (eds.) SCN 2004. LNCS, vol. 3352, pp. 120-133. Springer, Heidelberg (2005)

12. Camenisch, J., Lysyanskaya, A.: Dynamic Accumulators and Application to Efficient Revocation of Anonymous Credentials. In: Yung, M. (ed.) CRYPTO 2002. LNCS, vol. 2442, pp. 61-76. Springer, Heidelberg (2002)

13. Camenisch, J., Neven, G., Rückert, M.: Fully Anonymous Attribute Tokens from Lattices. In: Visconti, I., De Prisco, R. (eds.) SCN 2012. LNCS, vol. 7485, pp. 57-75. Springer, Heidelberg (2012) 
14. Cash, D., Hofheinz, D., Kiltz, E., Peikert, C.: Bonsai Trees, or How to Delegate a Lattice Basis. In: Gilbert, H. (ed.) EUROCRYPT 2010. LNCS, vol. 6110, pp. 523-552. Springer, Heidelberg (2010)

15. Chaum, D., van Heyst, E.: Group Signatures. In: Davies, D.W. (ed.) EUROCRYPT 1991. LNCS, vol. 547, pp. 257-265. Springer, Heidelberg (1991)

16. Chen, L., Pedersen, T.P.: New Group Signature Schemes (Extended Abstract). In: De Santis, A. (ed.) EUROCRYPT 1994. LNCS, vol. 950, pp. 171-181. Springer, Heidelberg (1995)

17. Fiat, A., Shamir, A.: How to Prove Yourself: Practical Solutions to Identification and Signature Problems. In: Odlyzko, A.M. (ed.) CRYPTO 1986. LNCS, vol. 263, pp. 186-194. Springer, Heidelberg (1987)

18. Gentry, C., Peikert, C., Vaikuntanathan, V.: Trapdoors for Hard Lattices and New Cryptographic Constructions. In: STOC, pp. 197-206. ACM (2008)

19. Gordon, S.D., Katz, J., Vaikuntanathan, V.: A Group Signature Scheme from Lattice Assumptions. In: Abe, M. (ed.) ASIACRYPT 2010. LNCS, vol. 6477, pp. 395-412. Springer, Heidelberg (2010)

20. Groth, J.: Fully Anonymous Group Signatures Without Random Oracles. In: Kurosawa, K. (ed.) ASIACRYPT 2007. LNCS, vol. 4833, pp. 164-180. Springer, Heidelberg (2007)

21. Kawachi, A., Tanaka, K., Xagawa, K.: Concurrently Secure Identification Schemes Based on the Worst-Case Hardness of Lattice Problems. In: Pieprzyk, J. (ed.) ASIACRYPT 2008. LNCS, vol. 5350, pp. 372-389. Springer, Heidelberg (2008)

22. Kiayias, A., Tsiounis, Y., Yung, M.: Traceable Signatures. In: Cachin, C., Camenisch, J.L. (eds.) EUROCRYPT 2004. LNCS, vol. 3027, pp. 571-589. Springer, Heidelberg (2004)

23. Laguillaumie, F., Langlois, A., Libert, B., Stehlé, D.: Lattice-Based Group Signatures with Logarithmic Signature Size. In: Sako, K., Sarkar, P. (eds.) ASIACRYPT 2013, Part II. LNCS, vol. 8270, pp. 41-61. Springer, Heidelberg (2013)

24. Langlois, A., Ling, S., Nguyen, K., Wang, H.: Lattice-based Group Signature Scheme with Verifier-local Revocation. Cryptology ePrint Archive, Report 2014/033 (2014), http://eprint.iacr.org/2014/033

25. Libert, B., Peters, T., Yung, M.: Group Signatures with Almost-for-Free Revocation. In: Safavi-Naini, R., Canetti, R. (eds.) CRYPTO 2012. LNCS, vol. 7417, pp. 571-589. Springer, Heidelberg (2012)

26. Libert, B., Vergnaud, D.: Group Signatures with Verifier-Local Revocation and Backward Unlinkability in the Standard Model. In: Garay, J.A., Miyaji, A., Otsuka, A. (eds.) CANS 2009. LNCS, vol. 5888, pp. 498-517. Springer, Heidelberg (2009)

27. Ling, S., Nguyen, K., Stehlé, D., Wang, H.: Improved Zero-Knowledge Proofs of Knowledge for the ISIS Problem, and Applications. In: Kurosawa, K., Hanaoka, G. (eds.) PKC 2013. LNCS, vol. 7778, pp. 107-124. Springer, Heidelberg (2013)

28. Lyubashevsky, V.: Lattice-Based Identification Schemes Secure Under Active Attacks. In: Cramer, R. (ed.) PKC 2008. LNCS, vol. 4939, pp. 162-179. Springer, Heidelberg (2008)

29. Lyubashevsky, V.: Lattice Signatures without Trapdoors. In: Pointcheval, D., Johansson, T. (eds.) EUROCRYPT 2012. LNCS, vol. 7237, pp. 738-755. Springer, Heidelberg (2012)

30. Micciancio, D., Peikert, C.: Trapdoors for Lattices: Simpler, Tighter, Faster, Smaller. In: Pointcheval, D., Johansson, T. (eds.) EUROCRYPT 2012. LNCS, vol. 7237, pp. 700-718. Springer, Heidelberg (2012)

31. Micciancio, D., Regev, O.: Lattice-based Cryptography. In: Post-Quantum Cryptography, pp. 147-191. Springer (2009) 
32. Micciancio, D., Vadhan, S.P.: Statistical Zero-Knowledge Proofs with Efficient Provers: Lattice Problems and More. In: Boneh, D. (ed.) CRYPTO 2003. LNCS, vol. 2729, pp. 282-298. Springer, Heidelberg (2003)

33. Nakanishi, T., Funabiki, N.: Verifier-Local Revocation Group Signature Schemes with Backward Unlinkability from Bilinear Maps. In: Roy, B. (ed.) ASIACRYPT 2005. LNCS, vol. 3788, pp. 533-548. Springer, Heidelberg (2005)

34. Nakanishi, T., Funabiki, N.: A Short Verifier-Local Revocation Group Signature Scheme with Backward Unlinkability. In: Yoshiura, H., Sakurai, K., Rannenberg, K., Murayama, Y., Kawamura, S.-I. (eds.) IWSEC 2006. LNCS, vol. 4266, pp. 17-32. Springer, Heidelberg (2006)

35. Peikert, C.: An Efficient and Parallel Gaussian Sampler for Lattices. In: Rabin, T. (ed.) CRYPTO 2010. LNCS, vol. 6223, pp. 80-97. Springer, Heidelberg (2010)

36. Peikert, C., Rosen, A.: Efficient Collision-Resistant Hashing from Worst-Case Assumptions on Cyclic Lattices. In: Halevi, S., Rabin, T. (eds.) TCC 2006. LNCS, vol. 3876, pp. 145-166. Springer, Heidelberg (2006)

37. Regev, O.: On Lattices, Learning with Errors, Random Linear Codes, and Cryptography. In: STOC, pp. 84-93. ACM (2005)

38. Rückert, M.: Adaptively Secure Identity-Based Identification from Lattices without Random Oracles. In: Garay, J.A., De Prisco, R. (eds.) SCN 2010. LNCS, vol. 6280, pp. 345-362. Springer, Heidelberg (2010)

39. Shor, P.W.: Polynomial-Time Algorithms for Prime Factorization and Discrete Logarithms on a Quantum Computer. SIAM Journal on Computing 26(5), 1484-1509 (1997)

40. Stern, J.: A New Paradigm for Public Key Identification. IEEE Transactions on Information Theory 42(6), 1757-1768 (1996) 